\title{
Análisis citogenético en Salminus brasiliensis (Pisces, Characidae) del Río Paraná, Corrientes, Argentina
}

\author{
Caramello, C.S.; Sánchez, S.; Jorge, L.C. \\ Instituto de Ictiología del Nordeste, Facultad de Ciencias Veterinarias, Universidad Nacional del Nordeste, \\ Sargento Cabral 2139, Corrientes (3400), Argentina. Tel/fax 03783-425753. E-mail: cynsolcar@hotmail.com
}

\begin{abstract}
Resumen
Caramello, C.S.; Sánchez, S.; Jorge, L.C.: Análisis citogenético en Salminus brasiliensis (Pisces, Characidae) del Río Paraná, Corrientes, Argentina. Rev. vet. 25: 1, 64-66, 2014. La especie Salminus brasiliensis es conocida vulgarmente con el nombre de "dorado". Se caracteriza por tener una amplia distribución en América del Sur. Los ejemplares de la población del Río Paraná se caracterizan por presentar un número diploide de 50 cromosomas. La fórmula cariotípica está constituida por 5 pares de cromosomas metacéntricos, 10 pares submetacéntricos y 10 pares subtelo- acrocéntricos, con un número fundamental de 80 en ambos sexos. No se observaron heteromorfismos cromosómicos relacionados con el sexo. La impregnación con nitrato de plata permitió observar la ocurrencia de NORs simples, localizadas en el brazo largo del par número 6 . El bandeo $\mathrm{C}$ reveló una gran cantidad de bloques de heterocromatina, presentes en la mayoría de los cromosomas, preferentemente situados en regiones centroméricas y pericentroméricas. El cariotípo de $S$. brasiliensis del Paraná Medio, Argentina, muestra con relación a individuos de la misma especie pertenecientes a las cuencas del Alto Paraná y San Francisco (Brasil), una notable similitud, evidenciando la ocurrencia de una estructura cariotípica muy conservada.
\end{abstract}

Palabras clave: Salminus brasiliensis, cariotipo, bandeo-C, NOR, Río Paraná.

\begin{abstract}
Caramello, C.S.; Sánchez, S.; Jorge, L.C.: Cytogenetic studies of Salminus brasiliensis (Pisces, Characidae) from Paraná River, Corrientes, Argentina. Rev. vet. 25: 1, 64-66, 2014. Salminus brasiliensis is commonly known as "dorado" ("golden"). It is widely distributed in South America. Paraná River's specimens are characterized by the presence of 50 chromosomes. Karyotipe formula is made up whith 5 metacentric, 10 submetacentric and 10 subtelo-acrocentric chromosomes pairs, with a fundamental number of 80 in both sexes. Chromosomal heteromorfism related whit sex was not detected. Single NORs were observed on the long arm in telomeric location of the submetacentric sixth pair. C-banding revealed a great amount or chromatin blocks in centromeric and pericentromeric regions of several chromosomes pairs. The karyotype of $S$. brasiliensis from Middle Paraná, Argentina, shows similarity considering individuals of the same species from the Upper Paraná and San Francisco basins (Brazil), thus determining a well conserved karyotypic structure feature.
\end{abstract}

Key words: Salminus brasiliensis, karyotype, C-banding, NOR, Paraná River.

\section{INTRODUCCIÓN}

La familia Characidae comprende una amplia diversidad de especies con marcada variedad morfológica. Los peces de esta familia se caracterizan por ser buenos nadadores y por vivir en ambientes muy diversos. Dentro de la familia Characidae, son reconocidos 170 géneros y 885 especies ${ }^{3,11}$. La subfamilia Salminae posee un único género Salminus, y tres especies $S$. hilarii, S. maxillosus y S. brasiliensis.

$S$. brasiliensis es conocido vulgarmente con el nombre de "dorado". Se caracteriza por tener una am-

Recibido: 19 diciembre 2013 / Aceptado: 4 febrero 2014 plia distribución en las reservas acuíferas de Sudamérica. Habita en las cuencas de los ríos Paraná, Paraguay, Bermejo, Dulce, Salí y Río de la Plata ${ }^{12}$.

Los especimenes de $S$. brasiliensis pueden alcanzar una longitud estándar superior a 1 metro. Presenta una coloración amarillo dorado, el dorso posee reflejos verdosos y el vientre es blanquecino. El cuerpo es robusto, ligeramente comprimido. La cabeza es grande y cónica. Los huesos operculares son grandes y poseen estrías. La boca es grande, con quijadas desiguales. Los dientes son cónicos. El dentario posee dos hileras de dientes. La aleta dorsal se encuentra localizada en la mitad del cuerpo y las aletas pectorales y ventrales están bien distanciadas entre sí. Estos peces son exce- 
lentes nadadores. Se alimentan de pequeños insectos y zooplancton en las primeras etapas de su desarrollo, luego se nutren de insectos mayores hasta convertirse finalmente en piscívoros ${ }^{1}$.

Debido a su importancia económica y a la escasa información que se tiene sobre la citogenética de $S$. brasiliensis, el objetivo del presente trabajo fue obtener datos genéticos de la especie, a través del análisis convencional de coloración con Giemsa, bandeos $\mathrm{C}$ y NOR en ejemplares provenientes del Río Paraná, Argentina.

\section{MATERIAL Y MÉTODOS}

Se analizaron 6 especimenes (tres hembras y tres machos) de $S$. brasiliensis, colectados en el Río Paraná. El sacrificio de los peces se realizó por sobredosis de anestésico (metasulfonato de tricaína MS222, Finquel ${ }^{\circledR}$ ).

Para el estudio de los cromosomas se utilizó la porción anterior del riñón ${ }^{10}$. Los cromosomas mitóticos se obtuvieron a través de la disociación del órgano en cubetas que contenían $10 \mathrm{ml}$ de solución de Hank's, posteriormente se continuó con los procedimientos de rutina ${ }^{5}$. Las regiones organizadoras de nucléolos (NOR) se identificaron por tinción con nitrato de plata (Ag-NOR) ${ }^{6}$. La heterocromatina constitutiva se localizó por el método convencional de bandeo $\mathrm{C}^{14}$.

Las metafases se fotografiaron con una cámara Digital Infinity 1 acoplada a un microscopio óptico Olympus BX41. Posteriormente se analizaron con el analiza- dor de imágenes Image ProPlus 5.1. En el cariotipo se organizaron los pares homólogos en orden decreciente de tamaño. Los cromosomas se clasificaron de acuerdo a su morfología y relación de brazos en metacéntricos (M), submetacéntricos (SM), subtelocéntricos (ST) y acrocéntricos $(\mathrm{A})^{7}$.

\section{RESULTADOS Y DISCUSIÓN}

El estudio citogenético de la especie reveló la presencia de un número diploide de 50 cromosomas. La fórmula cariotípica estaba constituida por $(10 \mathrm{M}+20$ $\mathrm{SM}+10 \mathrm{ST}-\mathrm{A})$. El NF= 80. No se detectó heteromorfismo relacionado con el sexo (Figura 1A). Datos similares fueron encontrados en ejemplares de esta especie provenientes de diferentes cuencas hidrográficas del Brasil $^{8,13}$.

Las regiones organizadoras de nucléolos evidenciadas por la tinción con nitrato de plata se localizaron en la región telomérica del brazo largo del sexto par cromosómico (SM) (Figura 1A). La ubicación y el número de NORs son constantes en todas las poblaciones de la especie estudiadas hasta el momento ${ }^{4,8,13}$.

A través del bandeo $\mathrm{C}$ se observó la presencia de bloques de heterocromatina constitutiva en las regiones centroméricas y pericentroméricas de la mayoría de los cromosomas del complemento (Figura 1B). Este patrón es semejante al descripto en especimenes de $S$. brasiliensis del territorio brasileño ${ }^{12}$. Los primeros pares

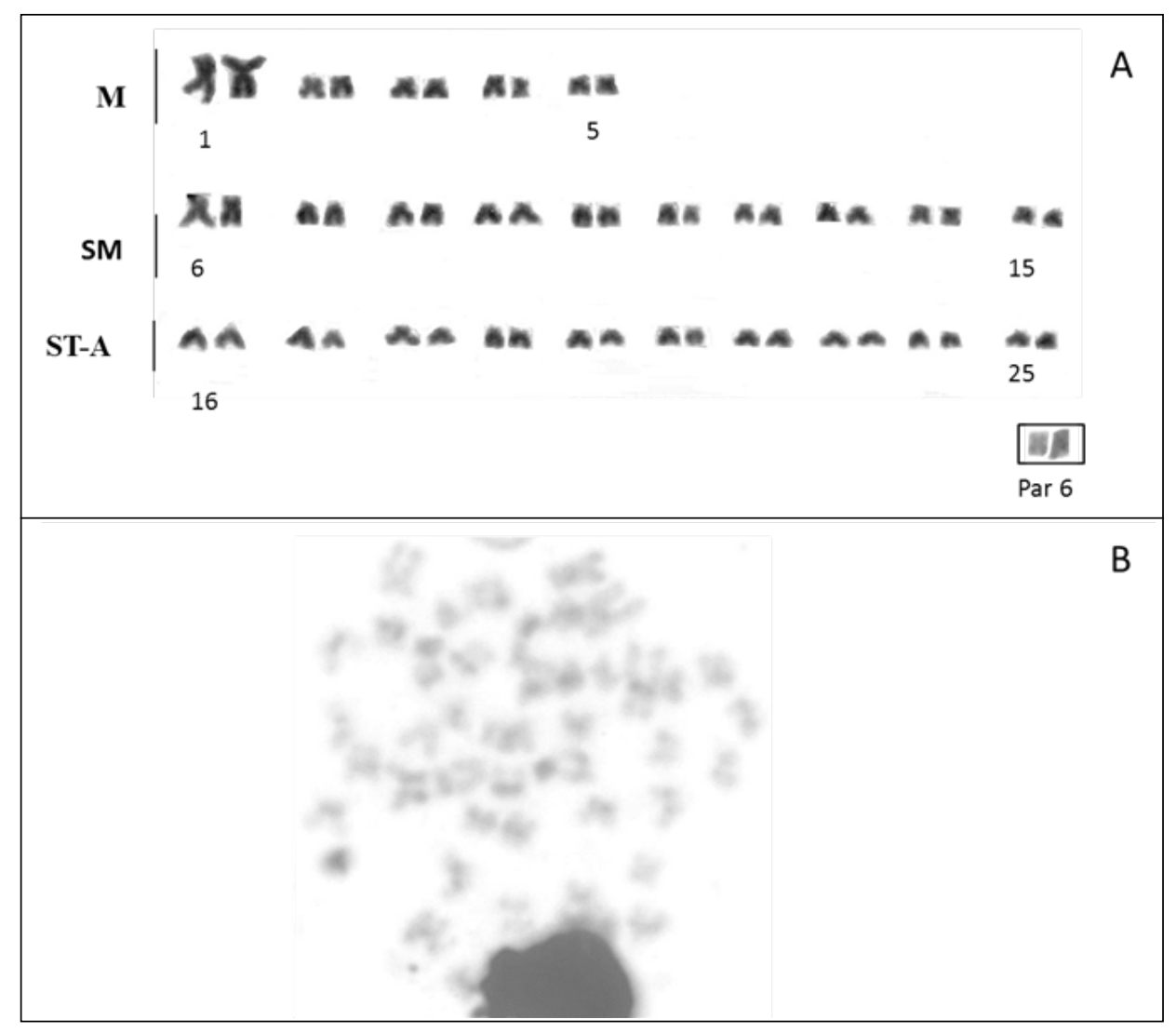

Figura 1. (A): cariotipo de Salminus brasiliensis con coloración convencional de Giemsa; en el recuadro se destacan los cromosomas portadores de la NOR (par 6). (B): bandeo C. 
de cromosomas metacéntricos y submetacéntricos del género Salminus, presentan una típica disposición de la heterocromatina constitutiva a nivel centromérico y pericentromérico, característica que también es compartida por otros carácidos ${ }^{2,9}$, condición que podría representar una sinapomorfía para la familia.

En conclusión, el cariotipo de Salminus brasiliensis del Paraná Medio, Argentina, muestra con relación a individuos de la misma especie pertenecientes a las cuencas del Alto Paraná y San Francisco (Brasil), una notable similitud, evidenciando la ocurrencia de una estructura cariotípica muy conservada.

\section{REFERENCIAS}

1. Casciotta J, Almirón A, Bechara J. 2003. Los peces de la laguna Iberá, Ed. Colección Universitaria, Buenos Aires, $131 \mathrm{p}$.

2. Daniel-Silva MF. 1996. Estudos citogenéticos comparativos em quatro espécies do gênero Astyanax (Pisces, Characidae). Master's Thesis, Instituto de Biociências, Universidade de São Paulo, Brasil.

3. Fink SV, Fink WL. 1981. Interrelationships of the ostariophysan fishes (Teleostei). Zool J Linn Soc Lond 72: $297-$ 353.

4. Foresti F, Almeida LF, Toledo AS. 1981. Polymorphic nature of nucleolus organizer regions in fishes. Cytogenet \& Cell Genet 31: 137-144.

5. Foresti F, Oliveira C, Almeida LF. 1993. A method for chromosome preparations from large specimens of fishes using in vitro short treatment with colchicine. Experientia 49: 810-813.
6. Howell WM, Black DA. 1980. Controlled silver-staining of nucleolus organizer regions with a protective colloidal developer: A 1-step method. Experientia 6: 1014-1015.

7. Levan A, Fredga K, Sandberg AA. 1964. Nomenclature for centromeric position on chromosomes. Hereditas 52: 201-220.

8. Marco DA. 1986. Estudos cromossômicos em peixes da subfamília Salmininae (Pisces, Characidae). Master's Thesis, Departamento de Ciências Biológicas, Universidade Federal de São Carlos, Brasil.

9. Margarido VP, Galetti PM. 1999. Heterochromatin patterns and karyotype relationships within and between the genera Brycon and Salminus (Pisces, Characidae). Genet Mol Biol 22: 357-361.

10. Moreira-Filho O, Bertollo LA. 1991. Extraction and use of the cephalic kidney for chromosome studies in small fish. Brazil J Genet 14: 1085-1090.

11. Nelson JS. 1994. Fishes of the world, 3rd edn., Wiley \& Sons, New York, $600 \mathrm{p}$.

12. Ringuelet RA, Aramburu RH, Aramburu AA. 1967. Los peces argentinos de agua dulce. Publ. Comisión de Investigaciones Científicas y Técnicas, Buenos Aires, 602 p.

13. Souza IL, Galián J, Santos-Silva LK, Venere PC, Moreira O. 2008. Molecular cytogenetics of Salminus fish (Characiformes) based on 5S and 18S rRNA genes hybridization, fluorochrome staining and C-banding. Science Direct Micron 39: 1036-1041.

14. Sumner AT. 1972. A simple technique for demonstrating centromeric heterochromatin. Experimental Cell Research 75: 304-306. 